\title{
Finite Element Analysis of the Microstructure-Strength Relationships of Metal Matrix Composites
}

\author{
Hai Qing
}

Received: 26 March 2014/Revised: 2 May 2014/Published online: 1 July 2014

(C) The Chinese Society for Metals and Springer-Verlag Berlin Heidelberg 2014

\begin{abstract}
The influence of the shape and spatial distribution of reinforced particles on strength and damage of metal matrix composite (MMC) is investigated through finite element method under uniaxial tensile, simple shear, biaxial tensile, as well as combined tensile/shear loadings. The particle shapes change randomly from circular to regular $n$-sided polygon ( $3 \leq n \leq 10)$; the particle alignments are determined through a sequentially random number stream and the particle locations are defined through the random sequential adsorption algorithm. The ductile failure in metal matrix and brittle failure in particles are described through damage models based on the stress triaxial indicator and maximum principal stress criterion, respectively, while the debonding behavior of interface between particles and matrix is simulated through cohesive elements. The simulation results show that, under different loadings, interface debonding is the dominated failure mechanism in MMCs and plastic deformation and ductile failure of matrix also play very important roles on the failure of MMCs.
\end{abstract}

\section{KEY WORDS: Metal matrix composites; Finite element analysis; Damage; Strength}

\section{Introduction}

Metal matrix composite is widely used in the fields of aerocraft [1], aeroengines [2], space component [3], aviation [4], and so on, due to its high specific stiffness and strength, high resistant to fire and radiation damage, etc. Generally, the macroscale properties of MMC can be achieved through experimental tests. In addition, theoretical micromechanical analysis $[5,6]$, and advanced numerical simulation $[7,8]$ are widely utilized to predict the macroscale properties from the known properties of the constituents. Compared to the experimental tests and theoretical micromechanical analysis, numerical simulation

Available online at http://link.springer.com/journal/40195

H. Qing $(\bowtie)$

State Key Laboratory of Mechanics and Control of Mechanical

Structures, Nanjing University of Aeronautics and Astronautics, Nanjing 210016, China

e-mail: qinghai@nuaa.edu.cn allows us to apply multiaxial loading condition easily and access the detailed microscale stress-strain fields and failure evolution during the loading process.

A number of numerical models have been developed to study the mechanical properties of metal matrix composite. Romanova et al. [9], Rasool and Bohm [10], and Garboczi and Douglas [11] studied the particle shape effects on the mechanical responses of MMCs. Yuan et al. [12], Ekici et al. [13], and Qing [14] studied the influence of reinforcement volume fractions on macroscale tensile stress/ strain curve of MMCs. Meijer et al. [15], Bouafia et al. [16], and Lu et al. [17] studied the influence of thermal residual stress on the stress-strain relationships of MMCs. Zhang et al. [18], Yang et al. [19], and Qing [20, 21] studied the influence of interphase on the mechanical properties of MMCs. Mishnaevsky et al. [22], Segurado et al. [23], and Ayyar and Chawla [24] investigated the effect of the inhomogeneous distribution of reinforced particles on the mechanical properties of MMCs. Xu and Palmiere [25], Prangnell [26], and Jia et al. [27] studied the macroscale stress-strain response of MMCs under 
compression loading. Xia and Curtin [28], Guo and Kang [29], and Kim et al. [30] investigated the fatigue failure behaviors of MMCs under cyclic loading.

In this paper, we study the influence of the loading conditions and microstructures of MMC on its mechanical properties. A Matlab program is developed to generate automatically the micromechanical finite element models with different shapes and spatial distributions of reinforced particles. The stress triaxial indicator and maximum principal stress based damage models are developed to simulate the ductile failure in metal matrix and brittle failure in particles, respectively. A series of numerical simulations are performed to study the macroscale stress-strain responses of MMCs and microscale damage initiation and evolution in MMCs under different loading conditions.

\section{Finite Element Model}

In this study, we utilize the random sequential adsorption algorithm [31,32] to generate the microstructure of composites. The microstructure of MMC is defined through the random number sequences with different random number generator seeds. Here, it is assumed that the unit cell (UC) of the MMC is a square with dimension $L_{0}$, and the particle number $n_{\mathrm{p}}$ and volume fraction of particles $v_{\mathrm{p}}$ are 100 and $15 \mathrm{vol} \%$, respectively. Meanwhile, it is also assumed that all the particles have a constant volume. Therefore, we can express the uniform particle volume $v_{0}$ as

$v_{0}=L_{0}^{2} v_{\mathrm{p}} / n_{\mathrm{p}}$.

Furthermore, it is assumed that the particle shapes vary from circular particle to regular $n$-sided polygons, where $n$ ranges from 3 to 10 . The distribution of particle shape is defined by a sequentially random number stream $N_{1}$ controlled by the random number generator seed $s_{1}$

$P_{i}=1+10 \times N_{1 i} \quad\left(i=1,2, \ldots, n_{\mathrm{p}}\right)$.

The shape of each particle is controlled by $P_{i}$ : if $P_{i}$ is less than 3, the $i$ th particle is circular particle; else if $P_{i}$ is not less than $n$ but less than $(n+1)$, the $i$ th particle is regular $n$-sided polygon. Meanwhile, another random number stream Rand $_{2}$ controlled by random number generator seed $s_{2}$ is applied to define the alignment of each particle. The locations of particle centers are produced independently and sequentially with two random number streams $\left(\operatorname{Rand}_{3}\right.$ and $\operatorname{Rand}_{4}$ ) controlled by two random number generator seeds $\left(s_{3}\right.$ and $\left.s_{4}\right)$. In this study, $s_{1}, s_{2}, s_{3}$, and $s_{4}$ obey the following relationships $s_{2}=s_{1}+1$, $s_{3}=s_{2}+1$, and $s_{4}=s_{3}+1$. In other words, the particle shapes, alignments, and locations are determined by $s_{1}$. The distances between the new particle and all existed particles must larger than a given distance related

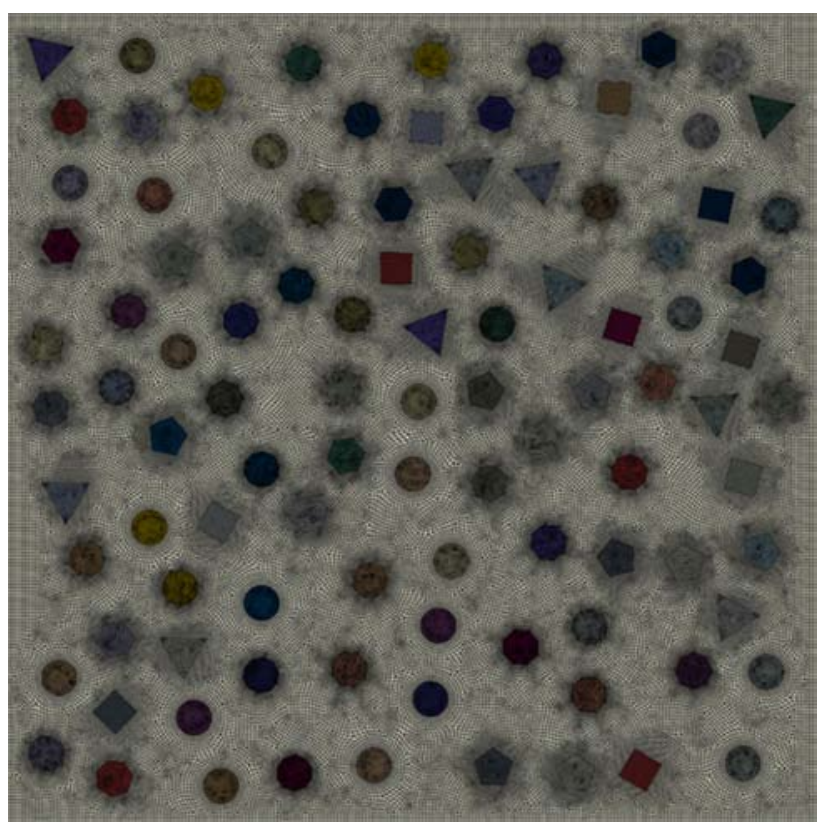

Fig. 1 Example of FE-mesh of microstructural model

to the radius of circular particle. Furthermore, the new particle should not be too close to the four borders.

A Matlab program is developed to realize the above algorithm. The Matlab code can generate a command file for the commercial software MSC/Patran. A 2D microstructural finite element model with pre-defined parameters (including one random number generator seed $s_{1}$ controlling the particle shape and spatial distribution in UC, the number and volume content of particle, mesh seeds, etc.) is generated through playing the command file with MSC/ Patran. The model is meshed through four-node quadrangular plane strain elements. Approximately 300 elements are used to represent each particle to ensure that the particle volume fraction in the discretized models is within $0.05 \%$ of the theoretical value. Figure 1 shows a FE-UC example generated through the program with $n_{\mathrm{p}}=100$ and $v_{\mathrm{p}}=15 \mathrm{vol} \%$. The different colors of particle represent the different mechanical properties.

The Young's modulus and Poisson's ratio of SiC particles are $410 \mathrm{GPa}$ and 0.14 , respectively. While the strengths of the $\mathrm{SiC}$ particles obey Weibull distribution with mean value of $\sigma_{\mathrm{a}}=500 \mathrm{MPa}$ [20] and shape parameter $m=9.62$ [33].

The elastic-plastic behavior of the aluminum alloy can be expressed as the Ramberg-Osgood model [34]

$\varepsilon=\frac{\sigma}{E_{\mathrm{m}}}+\alpha \frac{\sigma_{\mathrm{m}}}{E_{\mathrm{m}}}\left(\frac{\sigma}{\sigma_{\mathrm{m}}}\right)^{n_{\mathrm{m}}}$,

where Young's modulus $E_{\mathrm{m}}=69 \mathrm{GPa}$, and the three other parameters $\alpha=1, \sigma_{\mathrm{m}}=275 \mathrm{MPa}$, and $n_{\mathrm{m}}=11.66$, with 
the Poisson's ratio $v_{\mathrm{m}}=0.345$ and the strength $\sigma_{\mathrm{s}}=315 \mathrm{MPa}$. Isotropic hardening plasticity is applied to describe the Ramberg-Osgood model within Abaqus/ Standard. The yield stress $\sigma_{\mathrm{y}}$ and corresponding equivalent plastic strain $\varepsilon^{\mathrm{p}}$ can be expressed as

$\varepsilon^{\mathrm{p}}=\alpha \frac{\sigma_{\mathrm{m}}}{E_{\mathrm{m}}}\left(\frac{\sigma_{y}}{\sigma_{\mathrm{m}}}\right)^{n}$.

In this study, in order to describe the incremental damage indicator, we apply a triaxial factor $D$, which can be expressed as [35]

$D=\int_{0}^{\varepsilon_{\mathrm{p}}} \exp \left(\sigma_{\mathrm{H}} / \sigma_{\mathrm{V}}\right) \mathrm{d} \varepsilon_{\mathrm{p}}$.

In which, $\sigma_{\mathrm{H}}$ and $\sigma_{\mathrm{V}}$ are the hydrostatic stress and the von Mises stress, respectively, and $\varepsilon_{\mathrm{p}}$ is the accumulated plastic strain. The study [20] shows that $D=0.05$ is a suitable criterion for aluminum alloy.

2D cohesive element in term of traction-separation law is utilized to simulate the debonding behavior of interface. The initial stiffness $K_{i}$ of cohesive elements must be large enough to ensure the displacement continuity and avoid the modification of the stress and strain fields around the interface. Here, $K_{i}=10^{8} \mathrm{GPa}$ is adopted. The maximum stress criterion is applied to dictate the onset of interface debonding, and the initial damage strength of the cohesive element is assumed as

$S_{\text {int }}=\left(\sigma_{\mathrm{s}}+\sigma_{\mathrm{a}}\right) / 2$.

\section{Micromechanical Numerical Tests}

In order to study statistically the influence of microstructures of MMC on its mechanical properties, ten different micromechanical models $\left(n_{\mathrm{p}}=100\right.$ and $\left.v_{\mathrm{p}}=15 \mathrm{vol} \%\right)$ with different particle shapes, alignments, and arrangements are generated with different random number generator seeds $s_{1}$ following the methodology presented in the previous section. In order to eliminate the effect of UC borders, periodic boundary conditions are applied to the UC boundaries [14]. In this paper, engineering strain and stress are adopted to describe the macroscale strain and stress of UC.

\subsection{Uniaxial Tensile Loading and Simple Shear Loading}

In this subsection, the influence of the shapes, alignments, and arrangements of reinforced particles on the macroscale stress-strain curves of MMC and the microscale damage evolution in MMC is studied under uniaxial tensile and simple shear loading loadings. Figure 2 shows the simulation results of macroscale stress-strain curves of MMCs. From Fig. 2, one can see that the particle shape and spatial distribution in the micromechanical models plays little role in elastic properties of MMCs. However, the particle shape and spatial distribution in the micromechanical models does play a significant role in the maximum strengths and corresponding failure strain of composites of MMCs. In addition, MMC shows a stronger nonlinear response under simple shear loading than that under uniaxial tensile loading.

The failure element fractions in matrix, particle, and interface versus the macroscale strain with different particle shapes and spatial distributions are plotted in Figs. 3, 4, and 5 under uniaxial tensile and simple shear loadings, respectively. It can be seen that the tensile strains to initiate damage in matrix, particle, and interface are about $0.43 \%$, $0.17 \%$, and $0.08 \%$, respectively; the shear strains to initiate damage in matrix, particle, and interface are about $1.0 \%$, $0.24 \%$, and $0.15 \%$, respectively. Meanwhile, the evolutional trends of damage in matrix, particle, and interface are different under uniaxial tensile and simple shear loadings. The damage in matrix increases very fast almost at the final failure strain (final strain if we also look at Fig. 2), while damage in particles and interface firstly increases with the increase of the strain and increases very fast almost at the final failure strain. Furthermore, the damage in particles grows slowly with the loading process, and it grows a bit fast at the final failure strain of MMCs. The damage in interface grows fast and then a bit slowly with the loading process. Looking at the final failure elements, we can conclude that the interface debonding dominates the failure process, and MMCs are more effective under simple shear loading than under uniaxial tensile loading. Meanwhile, we can also notice that the failure matrix fractions are almost same level for both uniaxial tensile and simple shear loadings, while the failure particle and interface fractions under simple shear loading are higher than those under uniaxial tensile loading. The final failure element fractions of matrix are much higher than the final failure element fractions of particles under uniaxial tensile and simple shear loadings.

\subsection{Biaxial Tensile Loading}

In this subsection, the influence of the shapes, alignments, and arrangements of reinforced particles on the macroscale stress-strain curves of MMC and the microscale damage evolution in MMC is studied under the biaxial tensile loadings. The biaxial tensile loading is defined through the far field strain as $\varepsilon_{11}=k_{\mathrm{b}} \varepsilon_{22}$, where $k_{\mathrm{b}}$ is the biaxial factor and its value varies from 0.5 to 1.0 in this investigation. Figure 6 shows the simulation results of macroscale stress- 

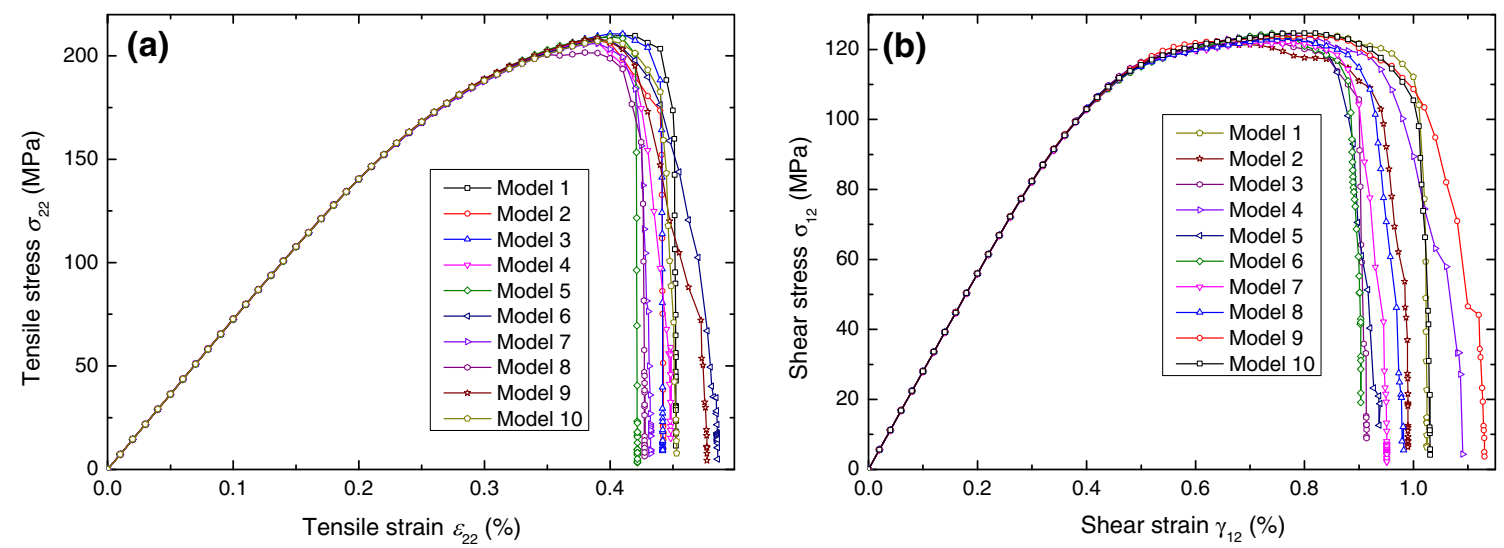

Fig. 2 Nominal stress-strain curves of different micromechanical models under uniaxial tensile a, simple shear loadings $\mathbf{b}$
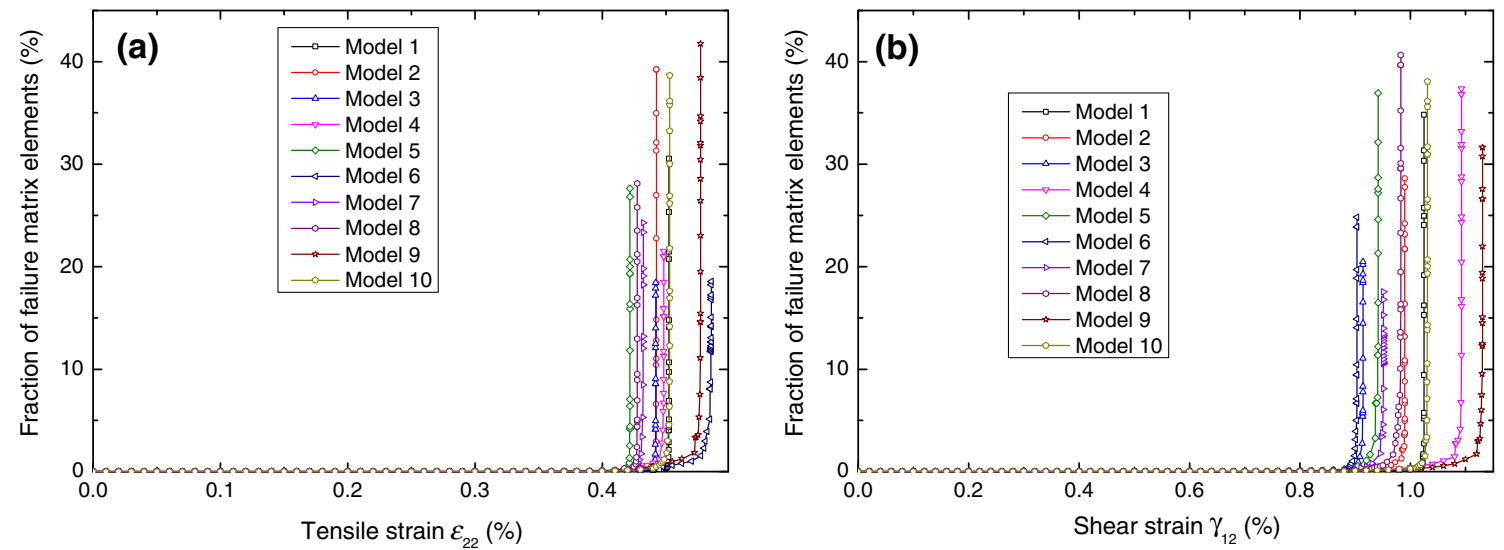

Fig. 3 Failure matrix fraction versus the nominal strain of different micromechanical models under uniaxial tensile a, simple shear loadings $\mathbf{b}$
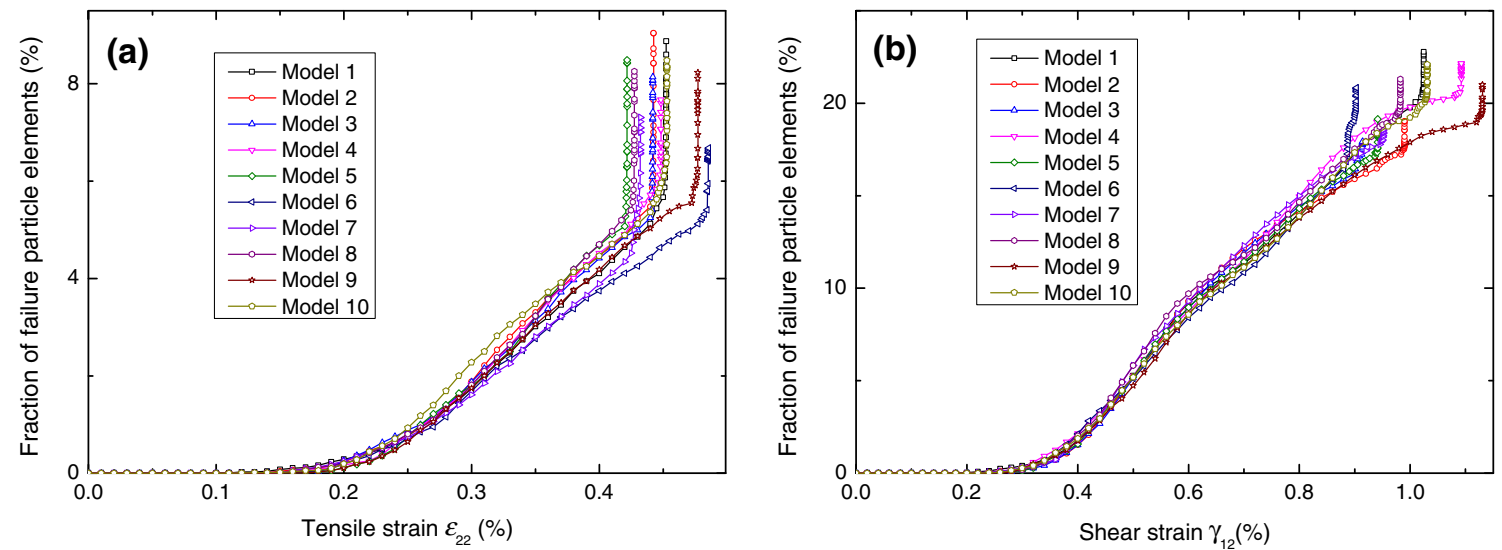

Fig. 4 Failure particle fraction versus the nominal strain of different micromechanical models under uniaxial tensile a, simple shear loadings $\mathbf{b}$

strain curves of MMCs. From Fig. 6, it can be seen again that the particle shapes and spatial distribution in the micromechanical models plays little role in elastic properties of MMCs. However, the particle shape and spatial distribution in the micromechanical models does play a significant role on the maximum strengths and corresponding failure strain of composites of MMCs. Comparing Fig. 6 with Fig. 2a, the nonlinear responses of MMCs under uniaxial tensile loading are much clearer and stronger than those under biaxial tensile loading. 

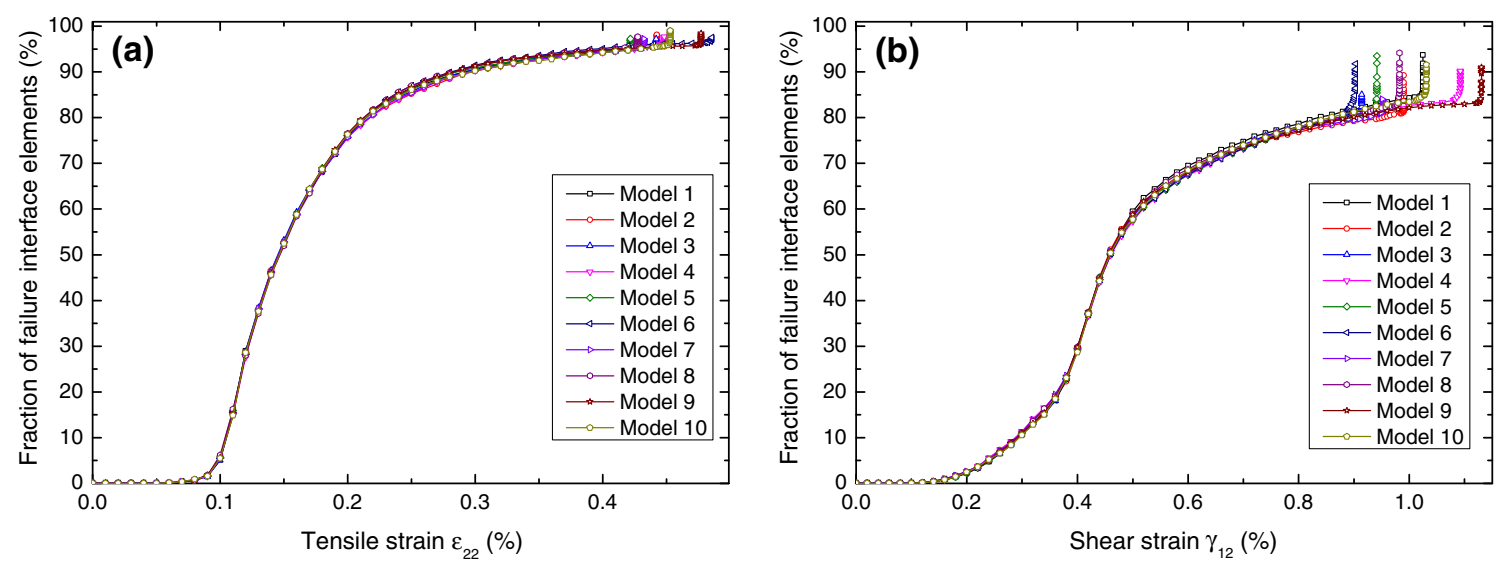

Fig. 5 Failure interface fraction versus the nominal strain of different micromechanical models under uniaxial tensile a, simple shear loadings $\mathbf{b}$
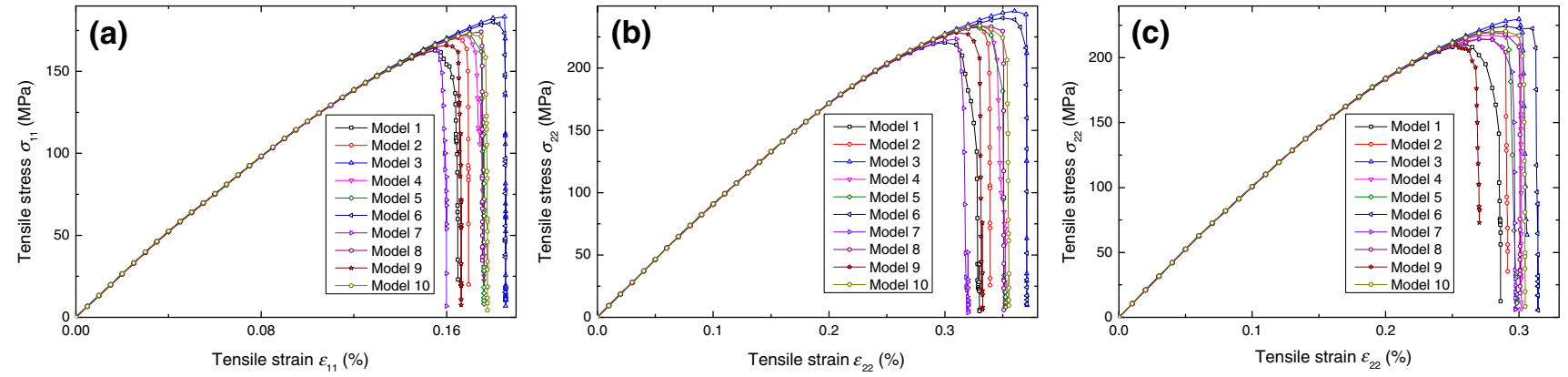

Fig. 6 Biaxial tensile nominal stress-strain curves of different micromechanical models: $\mathbf{a}, \mathbf{b} k_{\mathrm{b}}=0.5 ; \mathbf{c} k_{\mathrm{b}}=1.0$

The failure element fractions versus the nominal tensile strain in matrix, particle, and interface with different particle shapes and spatial distributions are plotted in Figs. 7, 8, and 9 under biaxial tensile loadings. Figure 7 shows that the strains to initiate damage in matrix are about $0.32 \%$ and $0.27 \%$ for $k_{\mathrm{b}}=0.5$ and $k_{\mathrm{b}}=1.0$, respectively; the damage in matrix grows very slowly till strain up to $0.32 \%$ and $0.27 \%$, respectively. Figure 8 shows that the strains to initiate damage in particles are about $0.18 \%$ and $0.15 \%$ for $k_{\mathrm{b}}=0.5$ and $k_{\mathrm{b}}=1.0$, respectively. The damage in particles grows very slowly to strain up to $0.18 \%$ and $0.15 \%$, respectively, and then grows a bit faster almost at final failure strains. Figure 9 shows the strains to initiate damage in interface are about $0.05 \%$ and $0.04 \%$ for $k_{\mathrm{b}}=0.5$ and $k_{\mathrm{b}}=1.0$, respectively. The damage in interface grows very fast within $0.3 \%$ strain, and almost all the interface elements are failed. Figures 7, 8, and 9 show that the failure element fractions in matrix, particle, and interface for $k_{\mathrm{b}}=1.0$ are higher than those for $k_{\mathrm{b}}=0.5$. In addition, comparing Figs. 7, 8, and 9 with Figs. 3, 4, and 5a, it can be seen that the final failure element fractions in matrix, particle, and interface under biaxial tensile loadings are higher than those under uniaxial tensile loading.

\subsection{Combined Tensile and Shear Loading}

In this subsection, the influence of the shapes, alignments, and arrangements of reinforced particles on the macroscale stress-strain curves of MMC and the microscale damage evolution in MMC is studied under combined tensile and shear loadings. The combined loading is defined through the far field strain as $\varepsilon_{22}=k_{\mathrm{c}} \gamma_{12}$, where $k_{\mathrm{c}}$ is the loading combination factor and its value varies from 0.5 to 1.0 to 2.0 in this investigation. Figures 10 and 11 show the simulation results of nominal stress-strain curves of MMCs. One can see that the particle shape and spatial distribution in the micromechanical models plays little role in the stress-strain relationships before damage initiates in MMC. However, the particle shape and spatial distribution in the micromechanical models does play a significant role on the plastic deformation of MMC, the maximum strengths, and the corresponding failure strain of composites. With the increase of $k_{\mathrm{c}}$, the shear strengths and corresponding shear strains decrease in Fig. 10, while the tensile strengths and corresponding tensile strains increase in Fig. 11.

The failure element fractions versus the nominal tensile strain in matrix, particle, and interface with different particle shapes and spatial distributions are, respectively, 

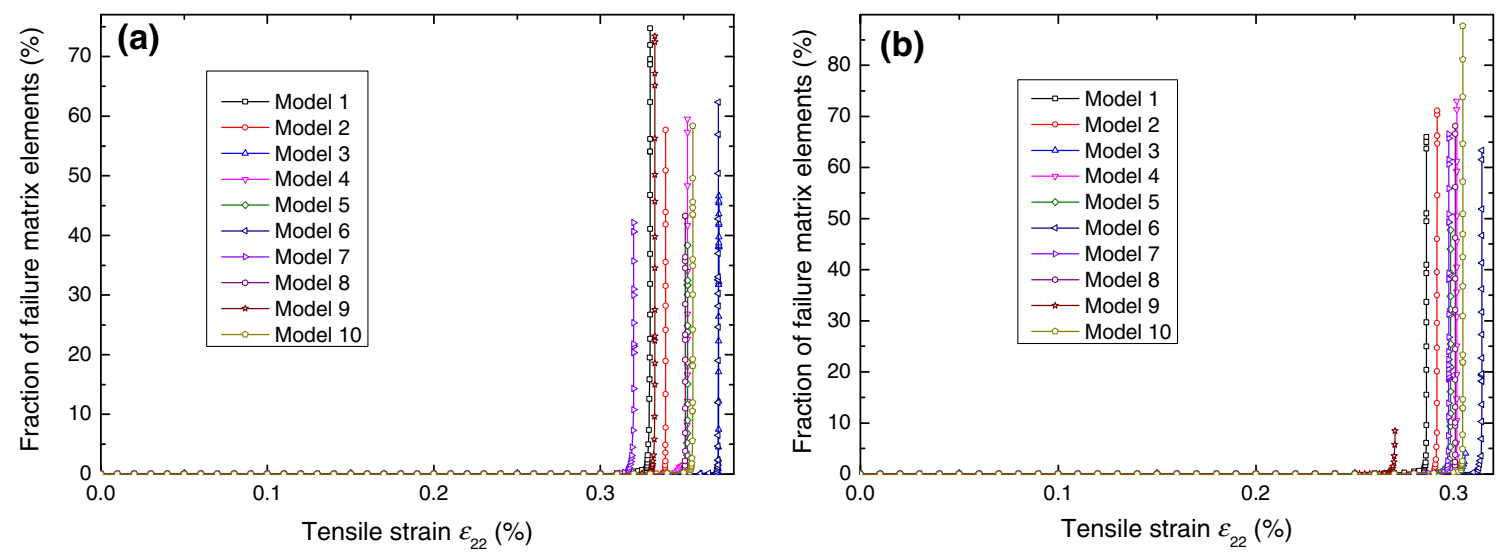

Fig. 7 Failure matrix fraction versus the nominal tensile strain of different micromechanical models for $k_{\mathrm{b}}=0.5 \mathbf{a}, k_{\mathrm{b}}=1.0 \mathbf{b}$
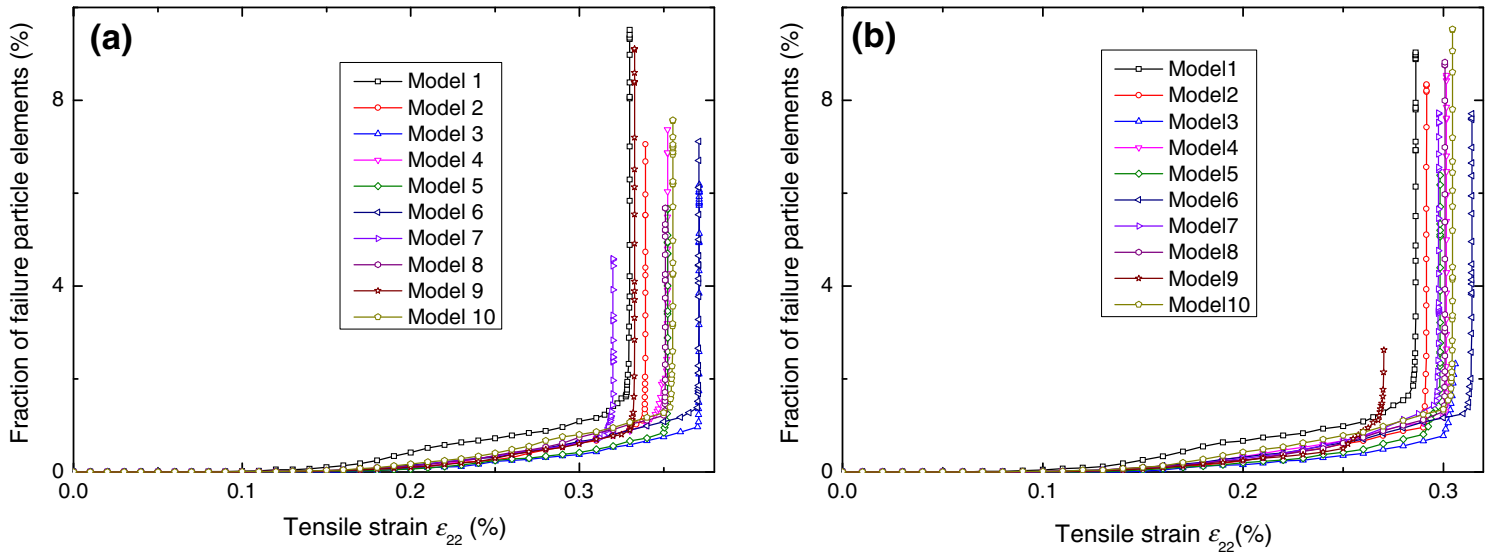

Fig. 8 Failure particle fraction versus the nominal tensile strain of different micromechanical models for $k_{\mathrm{b}}=0.5 \mathbf{a}, k_{\mathrm{b}}=1.0 \mathbf{b}$
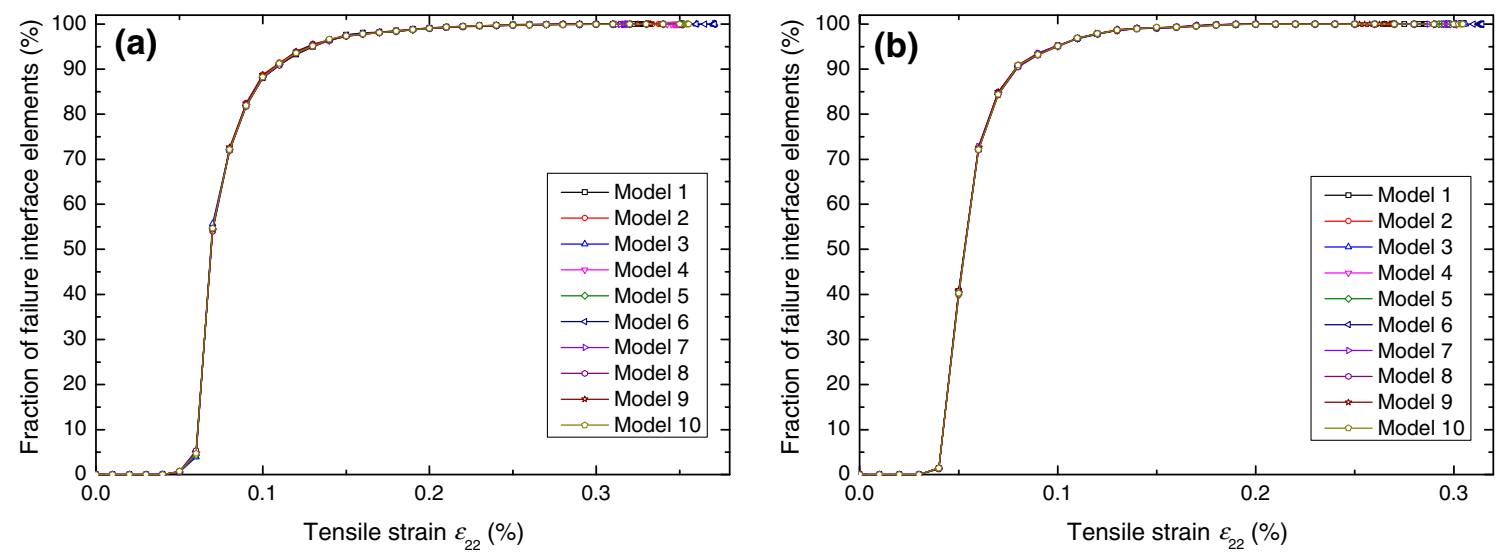

Fig. 9 Failure interface fraction versus the nominal tensile strain of different micromechanical models for $k_{\mathrm{b}}=0.5 \mathbf{a}, k_{\mathrm{b}}=1.0 \mathbf{b}$

plotted in Figs. 12, 13, and 14 under combined tensile and shear loadings. Figure 12 shows that the strains to initiate damage in matrix are about $0.27 \%, 0.34 \%$, and $0.37 \%$ for $k_{\mathrm{c}}$ varying from 0.5 to 1.0 to 2.0 . The damage in matrix grows very fast almost at the initial damage strains.
Figure 13 shows that the strains to initiate damage in particles are about $0.11 \%, 0.15 \%$, and $0.17 \%$ for $k_{\mathrm{c}}$ varying from 0.5 to 1.0 to 2.0. The damage in particles increases with the increase of tensile strain till final failure strain of matrix and then grows very fast almost at the strain level. 

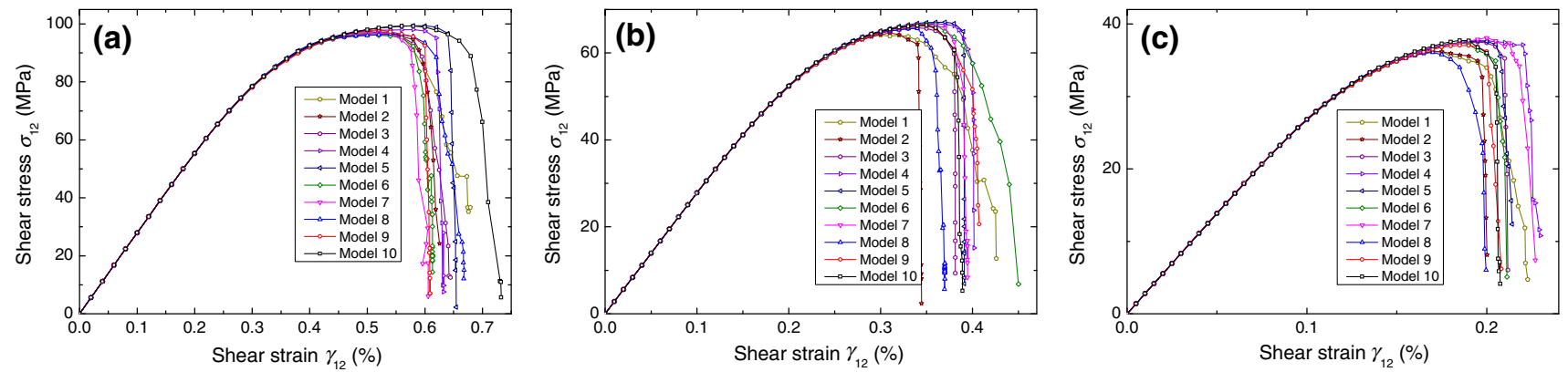

Fig. 10 Nominal shear stress-strain curves of different micromechanical models for $k_{\mathrm{c}}=0.5 \mathbf{a}, k_{\mathrm{c}}=1.0 \mathbf{b}, k_{\mathrm{c}}=2.0 \mathbf{c}$
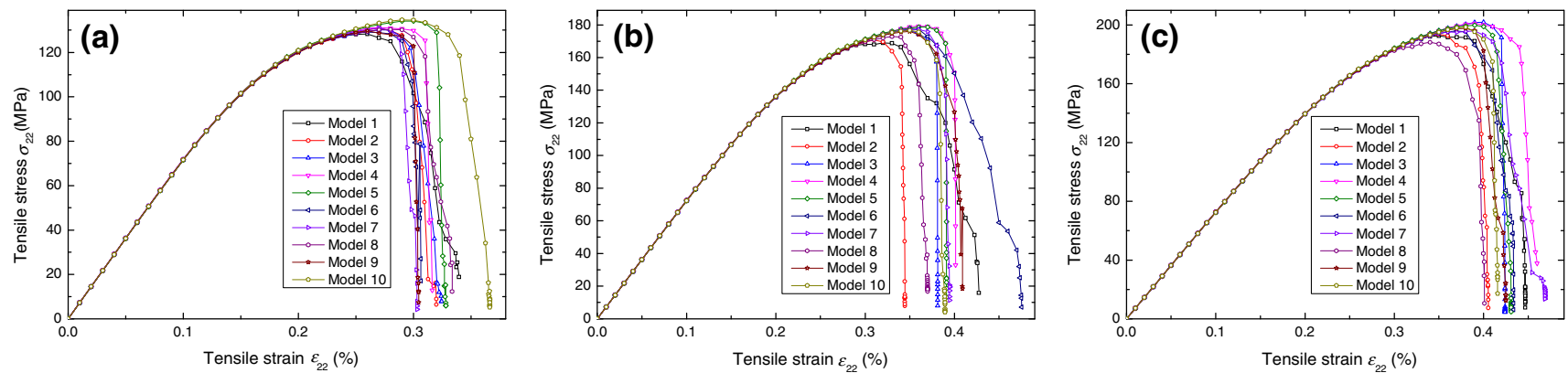

Fig. 11 Nominal tensile stress-strain curves of different micromechanical models for $k_{\mathrm{c}}=0.5 \mathbf{a}, k_{\mathrm{c}}=1.0 \mathbf{b}, k_{\mathrm{c}}=2.0 \mathbf{c}$
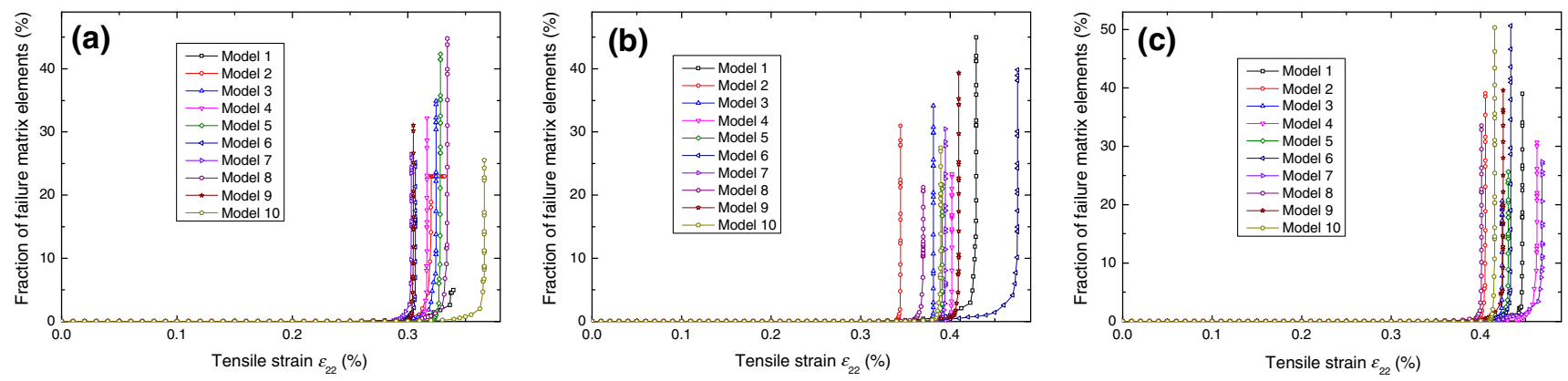

Fig. 12 Failure matrix fraction versus the nominal tensile strain of different micromechanical models for $k_{\mathrm{c}}=0.5 \mathbf{a}, k_{\mathrm{c}}=1.0 \mathbf{b}, k_{\mathrm{c}}=2.0 \mathbf{c}$
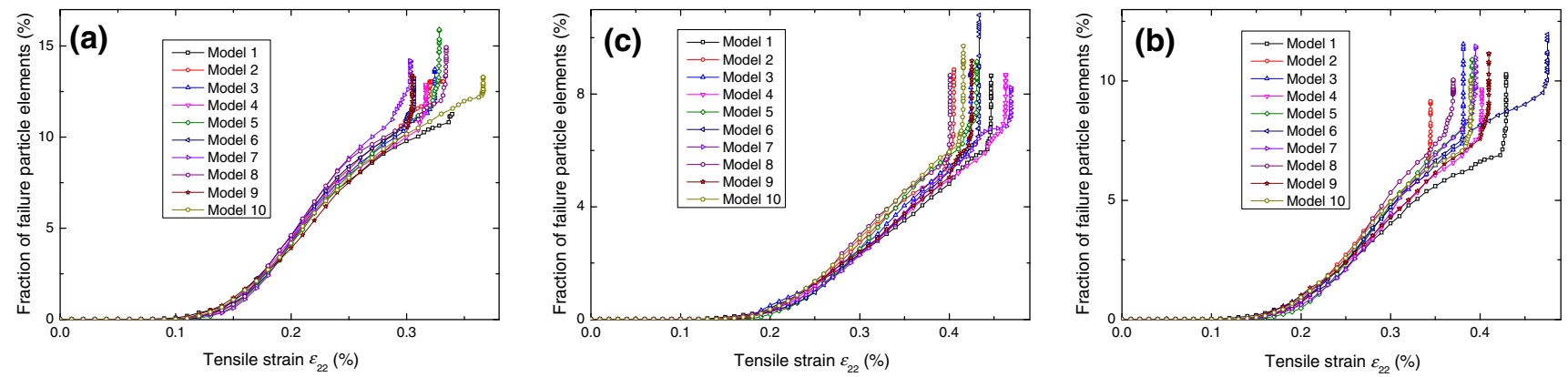

Fig. 13 Failure particle fraction versus the nominal tensile strain of different micromechanical models for $k_{\mathrm{c}}=0.5 \mathbf{a}, k_{\mathrm{c}}=1.0 \mathbf{b}, k_{\mathrm{c}}=2.0 \mathbf{c}$

Figure 14 shows that the strains to initiate damage in interface are about $0.05 \%, 0.07 \%$, and $0.08 \%$ for $k_{\mathrm{c}}$ varying from 0.5 to 1.0 to 2.0 . The damage in interface increases fast with the increase of tensile strain till initial damage strain of matrix and then grows very fast almost at the initial damage strains of matrix. 

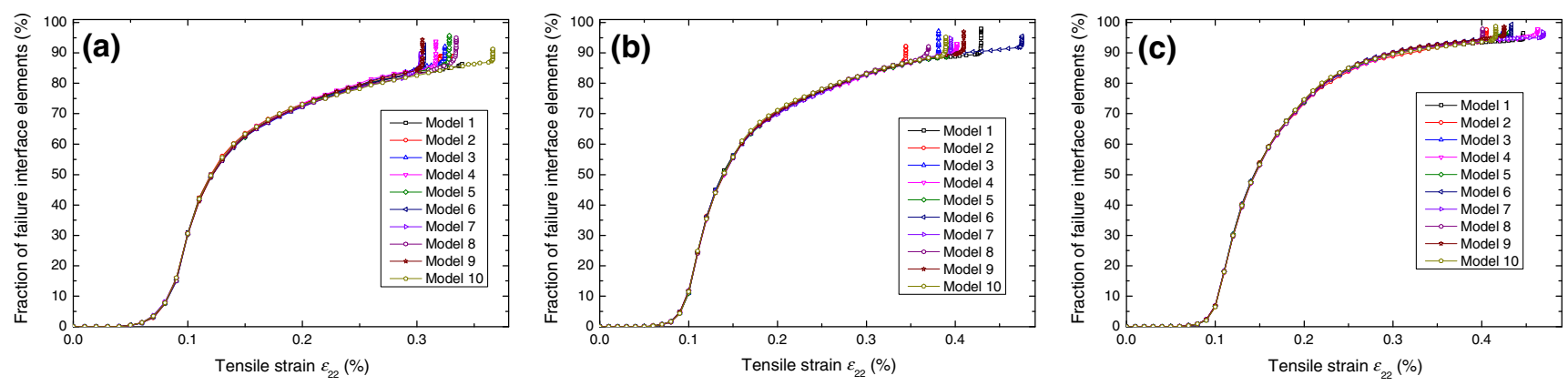

Fig. 14 Failure interface fraction versus the nominal tensile strain of different micromechanical models for $k_{\mathrm{c}}=0.5 \mathbf{a}, k_{\mathrm{c}}=1.0 \mathbf{b}, k_{\mathrm{c}}=2.0 \mathbf{c}$

Table 1 The failure strengths and corresponding strains for different particle shapes and arrangements under uniaxial and biaxial tensile loadings

\begin{tabular}{lllll}
\hline Loading type & $\varepsilon_{22}(\%)$ & $\varepsilon_{11}(\%)$ & $\sigma_{22}(\mathrm{MPa})$ & $207.490(2.405)$ \\
\hline Uniaxial & $0.396(0.009)$ & - & & $\sigma_{11}(\mathrm{MPa})$ \\
Biaxial & & & $232.680(6.980)$ & - \\
$k_{\mathrm{b}}=0.5$ & $0.327(0.017)$ & $0.169(0.01)$ & $218.195(5.719)$ & $171.830(6.154)$ \\
$k_{\mathrm{b}}=1.0$ & $0.277(0.014)$ & $0.278(0.014)$ & $220.040(5.708)$ \\
\hline
\end{tabular}

Numbers in the brackets indicate the standard deviations

Table 2 The failure strengths and corresponding strains for different particle shapes and arrangements under simple shear and combined tensile and shear loadings

\begin{tabular}{llllr}
\hline Loading type & $\varepsilon_{22}(\%)$ & $\gamma_{12}(\%)$ & $\sigma_{22}(\mathrm{MPa})$ & $\sigma_{12}(\mathrm{MPa})$ \\
\hline Simple shear & - & $0.748(0.039)$ & & $123.330(1.100)$ \\
Combined loading & & & & \\
$k_{\mathrm{c}}=0.5$ & $0.272(0.011)$ & $0.536(0.026)$ & $130.965(1.926)$ & $97.510(1.061)$ \\
$k_{\mathrm{c}}=1.0$ & $0.344(0.017)$ & $0.339(0.019)$ & $175.470(3.259)$ & $65.950(0.918)$ \\
$k_{\mathrm{c}}=2.0$ & $0.374(0.019)$ & $0.185(0.012)$ & $196.480(3.992)$ & $37.138(0.701)$ \\
\hline
\end{tabular}

Numbers in the brackets indicate the standard deviations

\subsection{Discussion}

Table 1 summarizes the average strengths and corresponding failure strains and their standard deviations of different particle shapes and arrangements under uni- and bi-axial tensile loading conditions. From Table 1, one can see that along y direction, the strength under uniaxial tensile loading is lower than that under biaxial tensile loadings, while the corresponding failure strain under uniaxial tensile loading is higher than that under biaxial tensile loadings. However, the tensile strength along $y$ direction for $k_{\mathrm{b}}=0.5$ is higher than that for $k_{\mathrm{b}}=1.0$. Along $x$ direction, both the strength and the corresponding failure strain increase with the increase of $k_{\mathrm{b}}$ from 0.5 to 1.0 .

Table 2 summarizes the average strengths and corresponding failure strains and their standard deviations of different particle shapes and arrangements under simple shear and combined tensile and shear loadings. From Table 2, it can be seen that along $y$ direction, the tensile strength and corresponding failure strain increase with the increase of $k_{\mathrm{c}}$. However, the shear strength and corresponding failure strain decrease as the loading condition changing from simple shear loading till the increase of $k_{\mathrm{c}}$.

\section{Conclusions}

A program is developed for the automatic generation of $2 \mathrm{D}$ micromechanical FE models of metal matrix composite containing reinforced particles with different shapes, alignments, and locations. A series of computational experiments are performed to study the influence of microstructure on composite stiffness and strength properties under different loading conditions. The following conclusions can be obtained: 
(1) Particle shapes and arrangements exert little elastic properties of MMCs. However, the particle shapes and arrangements of the micromechanical models play a significant role in the strengths and corresponding failure strains of MMCs.

(2) Among the three micromechanical failure mechanisms in the constituents of MMCs, interface debonding between particles and matrix dominates the failure of MMCs, and plastic deformation and ductile failure of matrix also play very important roles on the failure of MMCs.

(3) Under different loading conditions, interface debonding starts firstly, then particle brittle failure grows due to the Weibull distribution of particle strengths, matrix failure grows very fast almost without the further loading.

(4) Under uni- and bi-axial tensile loadings, failure strains decrease with the increase of $k_{\mathrm{b}}$. Under combined tensile and shear loadings and simple shear loading, one can observe a clear nonlinear response of MMC before its failure, and the nonlinear behaviors decrease with the increase of tensile loading part.

Acknowledgments The work was financially supported by the Fundamental Research Funds for the Central Universities (No. NE2014401) and a project funded by the Priority Academic Program Development of Jiangsu Higher Education Institutions

\section{References}

[1] E.A. Starke, J.T. Staley, Prog. Aerosp. Sci. 32, 131 (1996)

[2] J.A. Hooker, P.J. Doorbar, Mater. Sci. Technol. Lond. 16, 725 (2000)

[3] S. Rawal, JOM 53, 14 (2001)

[4] I.N. Orbulov, K. Májlinger, Mater. Des. 49, 1 (2013)

[5] S. Nemat-Nasser, M. Hori, in Micromechanics: Overall Properties of Heterogeneous Materials, 2nd edn. (Elsevier, Amsterdam, 1994)

[6] S. Torquato, in Random Heterogeneous Materials, (Springer, Heidelberg, 2006)
[7] L. Mishnaevsky, in Computational Mesomechanics of Composites: Numerical Analysis of the Effect of Microstructures of Composites of Strength and Damage Resistance, (Wiley, Hoboken, 2007)

[8] T.I. Zohdi, P. Wriggers, in an Introduction to Computational Micromechanics, (Springer, Heidelberg, 2008)

[9] V.A. Romanova, R.R. Balokhonov, S. Schmauder, Acta Mater. 57, 97 (2009)

[10] A. Rasool, H.J. Bohm, Int. J. Eng. Sci. 58, 21 (2012)

[11] E.J. Garboczi, J.F. Douglas, Mech. Mater. 51, 53 (2012)

[12] M.N. Yuan, Y.Q. Yang, C. Li, P.Y. Heng, L.Z. Li, Mater. Des. 38, 1 (2012)

[13] R. Ekici, M.K. Apalak, M. Yildirim, F. Nair, F. Mater. Des. 31, $2818(2010)$

[14] H. Qing, Mater. Des. 44, 446 (2013)

[15] G. Meijer, F. Ellyin, Z. Xia, Compos. Pt. B Eng. 31, 29 (2000)

[16] F. Bouafia, B. Serier, B.A.B. Bouiadjra, Comput. Mater. Sci. 54, 195 (2012)

[17] J. Lu, B. Miege, J.F. Flavenot, S. Thery, J. Compos. Technol. Res. 12, 232 (1990)

[18] W.X. Zhang, L.X. Li, T.J. Wang, Comput. Mater. Sci. 41, 145 (2007)

[19] X. Yang, G.Q. Wu, W. Sha, Q.Q. Zhang, Z. Huang, Compos. Pt. A. Appl. Sci. Manuf. 43, 363 (2012)

[20] H. Qing, Mater. Des. 51, 438 (2013)

[21] H. Qing, Comput. Mater. Sci. 89, 102 (2014)

[22] L. Mishnaevsky, K. Derrien, D. Baptiste, Compos. Sci. Technol. 64, 1805 (2004)

[23] J. Segurado, C. Gonzalez, J. Llorca, Acta Mater. 51, 2355 (2003)

[24] A. Ayyar, N. Chawla, Acta Mater. 55, 6064 (2007)

[25] H. Xu, E.J. Palmiere, Compos. Part A. 30, 203 (1999)

[26] P.B. Prangnell, S.J. Barnes, S.M. Roberts, P.J. Withers, Mater. Sci. Eng. A 220, 41 (1996)

[27] N. Jia, F. Roters, P. Eisenlohr, D. Raabe, X. Zhao, Acta Mater. 61, 4591 (2013)

[28] Z.H. Xia, W.A. Curtin, Acta Mater. 49, 1633 (2001)

[29] S.J. Guo, G.Z. Kang, Acta Metall. Sin. 42, 1051 (2006). (in Chinese)

[30] S.B. Kim, D.A. Koss, D.A. Gerard, Mat. Sci. Eng. A 277, 123 (2000)

[31] H.W. Wang, H.W. Zhou, R.D. Peng, L. Mishnaevsky, Compos. Sci. Technol. 71, 980 (2011)

[32] B. Zhang, Z. Yang, X. Sun, Z. Tang, Comput. Mater. Sci. 49, 645 (2010)

[33] C. Lu, R. Danzer, F.D. Fischer, Phys. Rev. E 65, 067102 (2002)

[34] G. Bao, Z. Lin, Acta Mater. 44, 1011 (1996)

[35] J.R. Rice, D.M. Tracey, J. Mech. Phys. Solids 17, 201 (1969) 